\title{
Antifibrotic effects of PDE4 blockade?
}

PDE4
inhibitors such
as apremilast
might ... have
potential in the
treatment of
fibrosis in SSc

New research published in Annals of the Rheumatic Diseases suggests inhibition of phosphodiesterase 4 (PDE4) could have disease-modifying antifibrotic effects in systemic sclerosis (SSc), particularly in those patients with inflammation-driven fibrosis. In preclinical models of SSc, PDE4 blockade prevented progression of chronic fibrosis and also reversed established fibrosis by reducing inflammatory cell activity and inhibiting the release of profibrotic cytokines from M2 macrophages.

Treatment with the PDE4 inhibitor rolipram hampered the development of skin fibrosis in bleomycin-challenged mice in a dose-dependent manner. Skin thickness, amount of fibrotic tissue and myofibroblast numbers were substantially lower in mice treated with rolipram in comparison with vehicle-treated mice. Notably, leukocyte

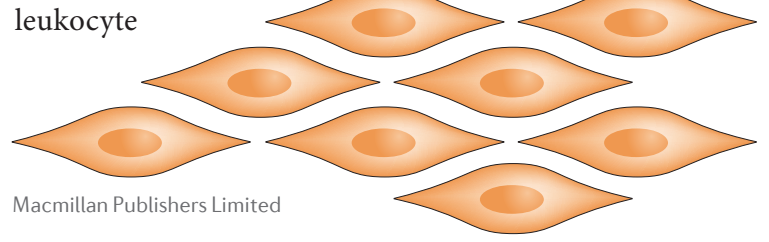

infiltration in lesional skin was markedly reduced by rolipram treatment, but fibroblasts were not directly affected.

In peripheral blood monocytes isolated from healthy volunteers and patients with diffuse-cutaneous SSc, PDE4 blockade with rolipram inhibited the differentiation of monocytes into an alternatively activated M2 macrophage phenotype, but not into a classically activated M1 phenotype. Rolipram treatment also reduced mRNA expression of the profibrotic cytokines IL6, IL13, TGFB1 and TGFB2 in M2 macrophages (but not M1 macrophages), as well as secretion of IL-6. Consistent with these in vitro findings, numbers of M2 macrophages and tissue levels of IL-6 were reduced in skin sections from mice with bleomycin-induced fibrosis treated with rolipram.

PDE4 blockade was also shown to have antifibrotic effects in mice with established bleomycin-induced fibrosis. In this model, treatment with the PDE4 inhibitor apremilast prevented progression of chronic fibrosis and also induced regression of established fibrosis. Additionally, leukocyte infiltration, M2 macrophage differentiation and tissue levels of IL- 6 were reduced after apremilast treatment. Pharmacological PDE4 blockade also showed antifibrotic effects in the topoisomerase I mouse model of fibrosis and in murine sclerodermatous graft-versus-host disease, a model of diffuse-cutaneous SSc fibrosis.

Notably, apremilast is already clinically approved for the treatment of psoriasis and psoriatic arthritis. The findings of the current study suggest that PDE4 inhibitors such as apremilast might also have potential in the treatment of fibrosis in SSc, perhaps in early-stage SSc, which is characterized by inflammatory infiltrates, or in inflammatory subtypes of SSc in particular.

Sarah Onuora

ORIGINAL ARTICLE Maier, C. et al. Inhibition of phosphodiesterase 4 (PDE4) reduces dermal fibrosis by interfering with the release of interleukin- 6 from M2 macrophages. Ann. Rheum. Dis. http://dx.doi. org/10.1136/annrheumdis-2016-210189 (2017) 